Supporting information

\title{
Mesoporous Crystalline Niobium Oxide with High Surface Area: A Solid Acid Catalyst for Alkyne Hydration
}

Dinithi Rathnayake ${ }^{a}$, Inosh Perera ${ }^{\mathrm{a}}$, Alireza Shirazi-Amin ${ }^{\mathrm{a}}$, Peter Kerns ${ }^{\mathrm{a}}$, Shanka Dissanayake ${ }^{\mathrm{a}}$, and Steven L. Suib ${ }^{* a b}$

a Department of Chemistry, University of Connecticut, 55 North Eagleville Road, Unit 3060,

Storrs, CT 06269, USA.

${ }^{b}$ Institute of Material Science, University of Connecticut, 97 North Eagleville Road, Unit 3136, Storrs, CT 06269, USA.

*steven.suib@uconn.edu 


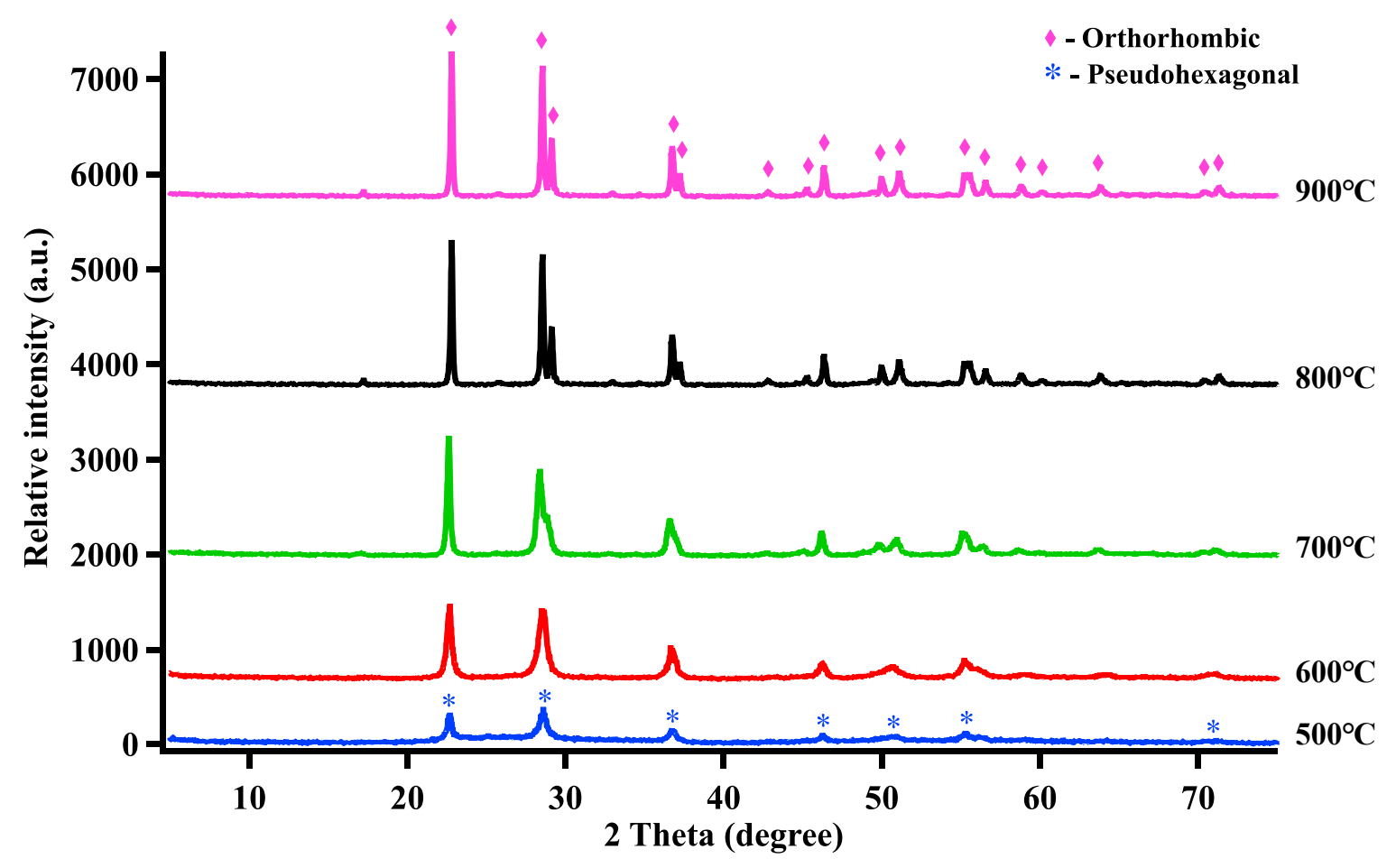

Figure S1. XRD patterns of $\mathrm{Nb}_{2} \mathrm{O}_{5}$ calcined at different temperatures.

Table S1. Crystallite size at calculation at different temperatures by Halder-Wagner method.

\begin{tabular}{|c|c|c|}
\hline Calcination temperature $\left({ }^{\circ} \mathrm{C}\right)$ & Crystal structure & Crystallite size $(\mathrm{nm})$ \\
\hline 500 & Pseudohexagonal & 19.5 \\
600 & Pseudohexagonal & 20.3 \\
700 & Orthorhombic & 22.7 \\
800 & Orthorhombic & 39.2 \\
900 & Orthorhombic & 63.1 \\
\hline
\end{tabular}


Table S2. Pore data obtained for the synthesized materials.

\begin{tabular}{|c|c|c|c|}
\hline Material & Surface area $\left(\mathrm{m}^{2} / \mathrm{g}\right)$ & Pore volume $(\mathrm{cc} / \mathrm{g})$ & Pore diameter $(\mathrm{nm})$ \\
\hline $\mathrm{Nb}_{2} \mathrm{O}_{5}-1 \mathrm{~h}$ & 155 & 0.29 & 4.9 \\
$\mathrm{Nb}_{2} \mathrm{O}_{5}-3 \mathrm{~h}$ & 127 & 0.25 & 5.6 \\
\hline
\end{tabular}

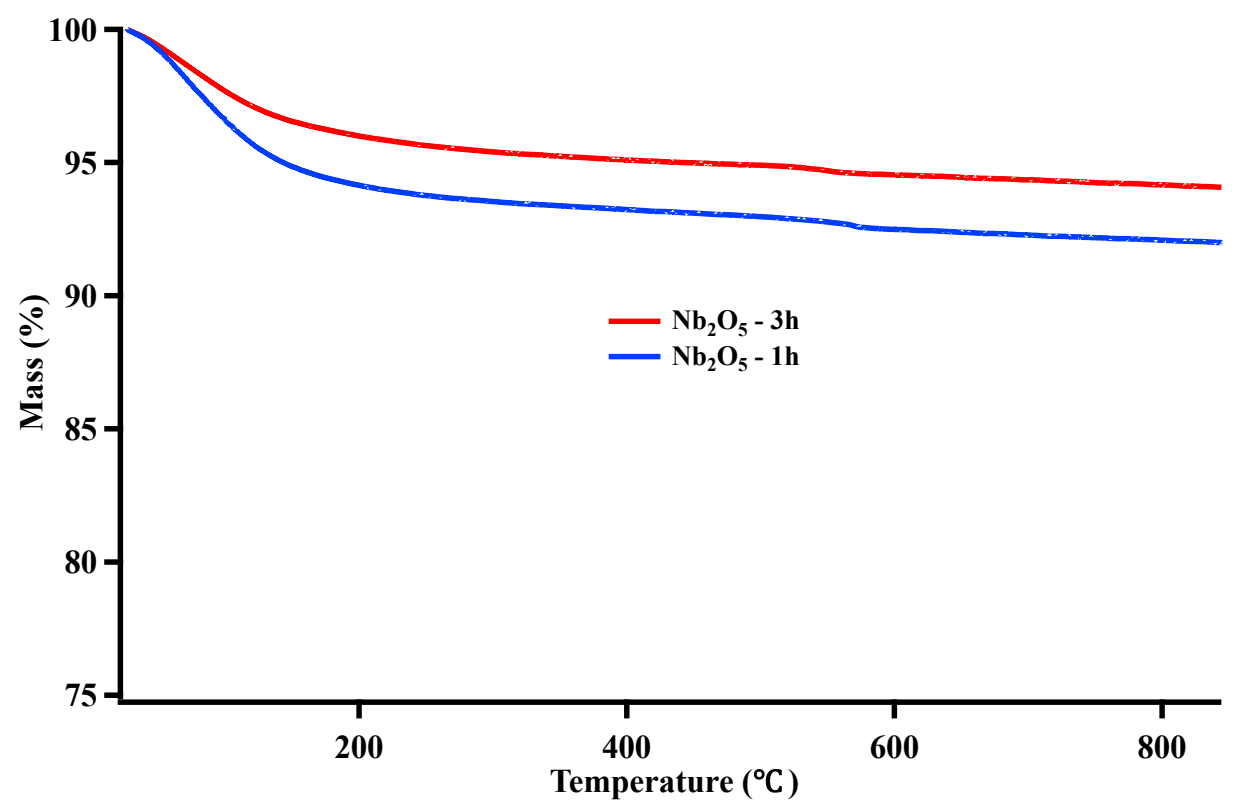

Figure S2. TGA curves of $\mathrm{Nb}_{2} \mathrm{O}_{5}-1 \mathrm{~h}$ and $\mathrm{Nb}_{2} \mathrm{O}_{5}-3 \mathrm{~h}$. 
(a)
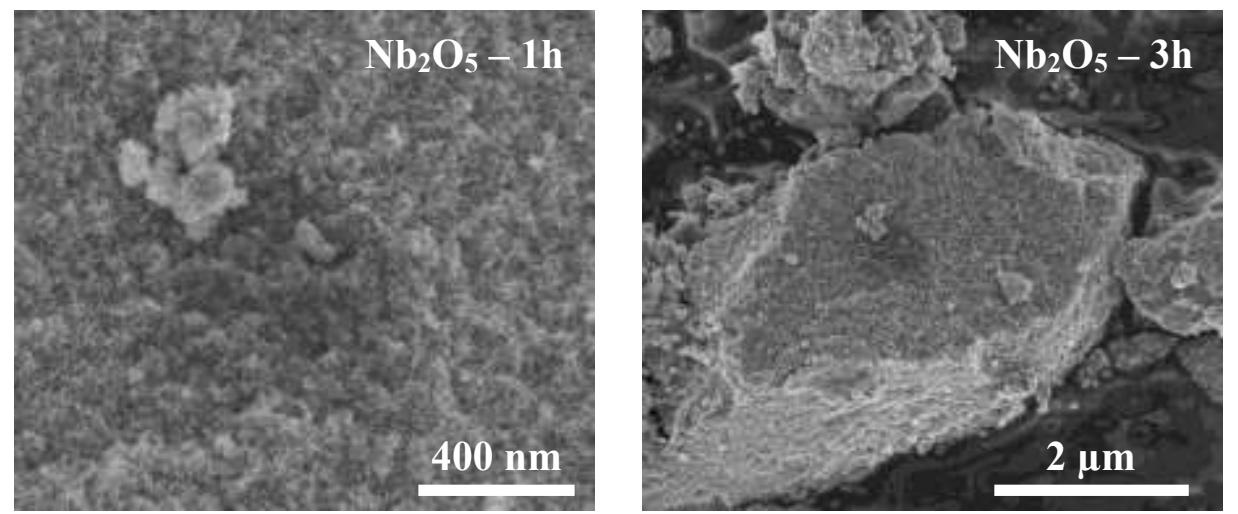

(b)
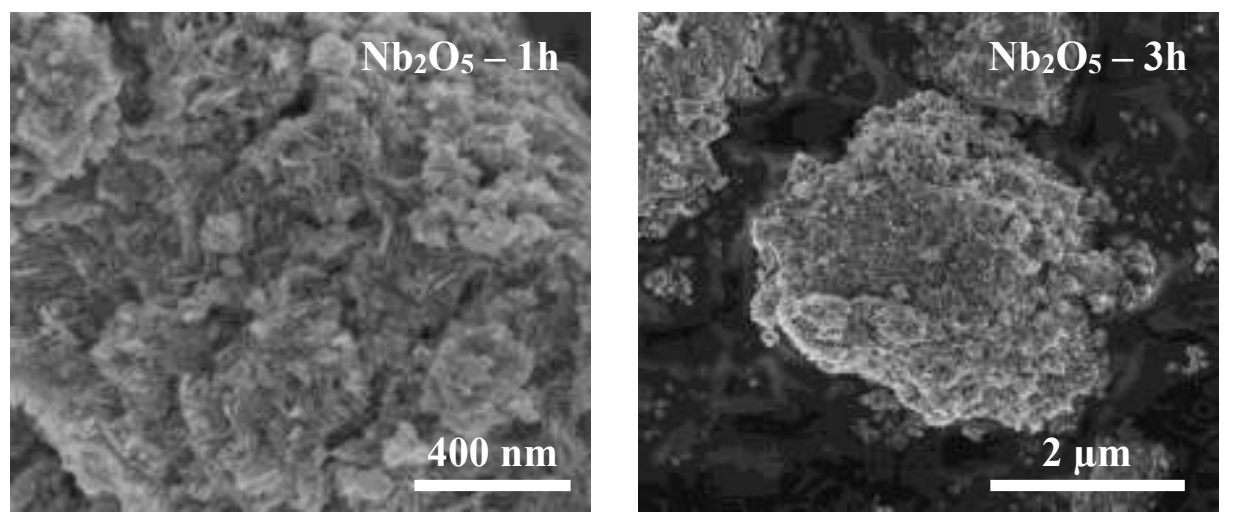

Figure S3. SEM images of (a) $\mathrm{Nb}_{2} \mathrm{O}_{5}-1 \mathrm{~h}$, and $\mathrm{Nb}_{2} \mathrm{O}_{5}-3 \mathrm{~h}$. 

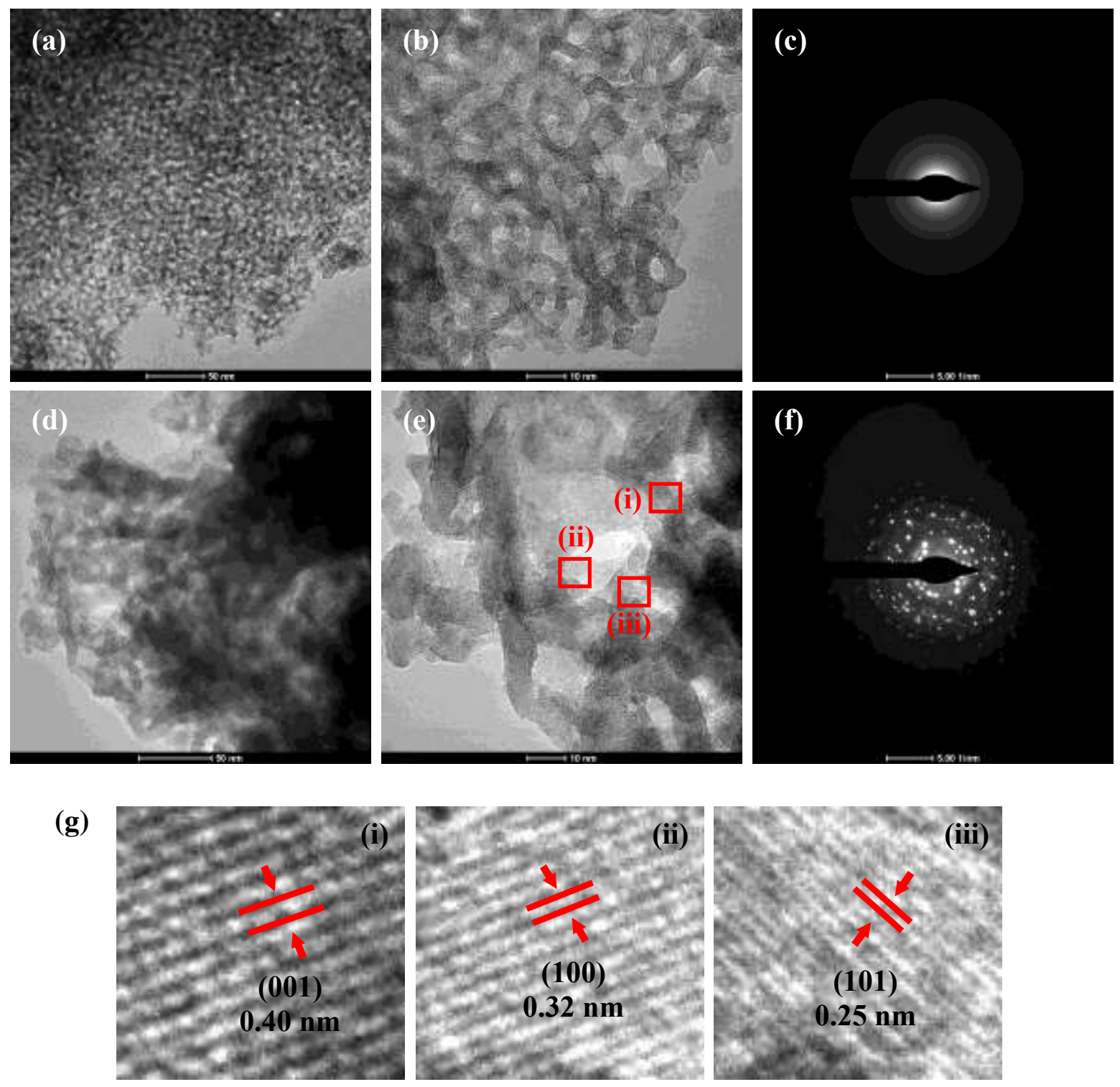

Figure 4. (a,b) HR-TEM images of $\mathrm{Nb}_{2} \mathrm{O}_{5}-1 \mathrm{~h}$, (c) SAED pattern of $\mathrm{Nb}_{2} \mathrm{O}_{5}-1 \mathrm{~h}$, (d,e) HR-TEM images of $\mathrm{Nb}_{2} \mathrm{O}_{5}-3 \mathrm{~h}$, (f) SAED pattern of $\mathrm{Nb}_{2} \mathrm{O}_{5}-3 \mathrm{~h}$, and (g) enlarged images of lattice fringes in $\mathrm{Nb}_{2} \mathrm{O}_{5}-3 \mathrm{~h}$. 
(a)

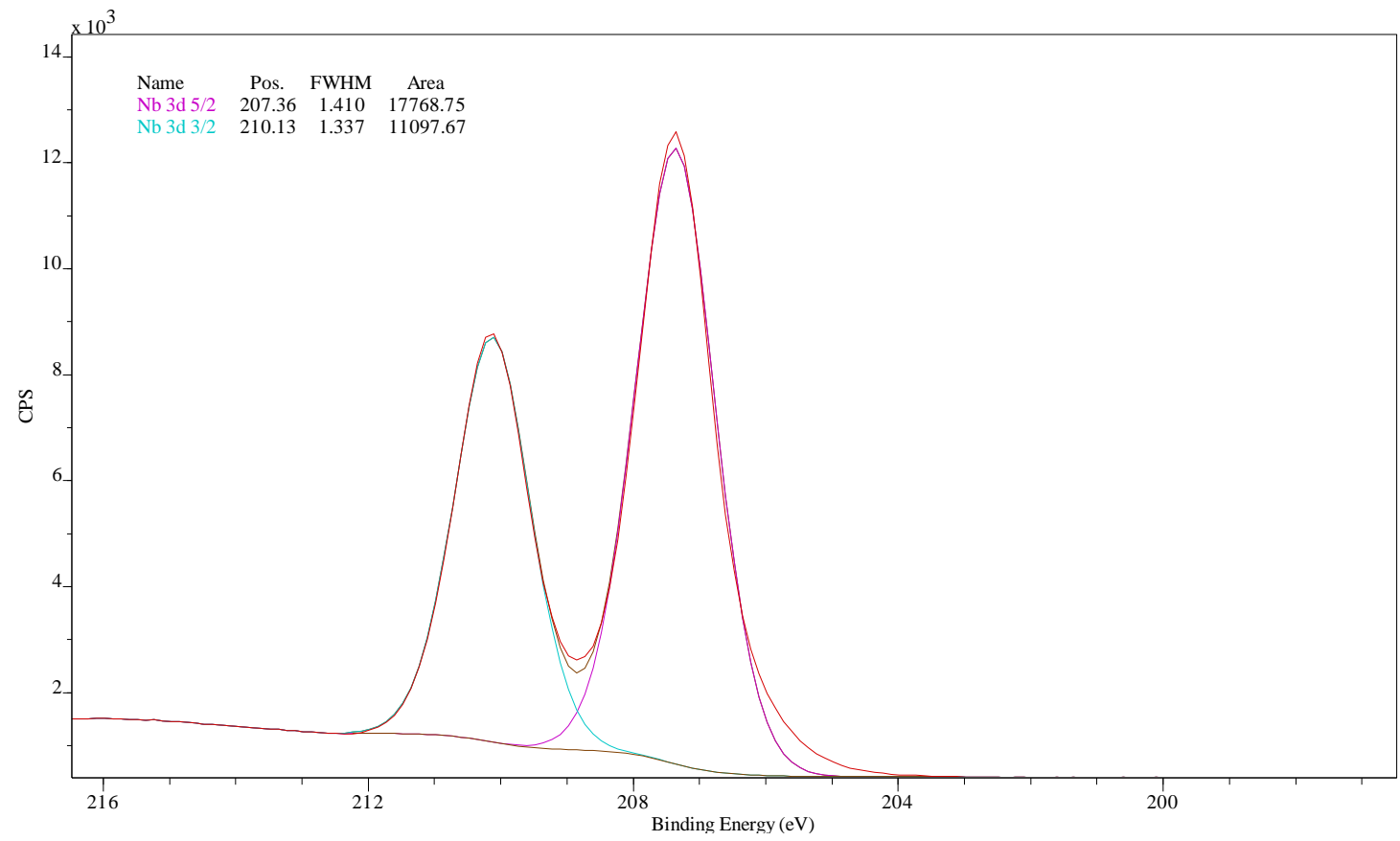

(b)

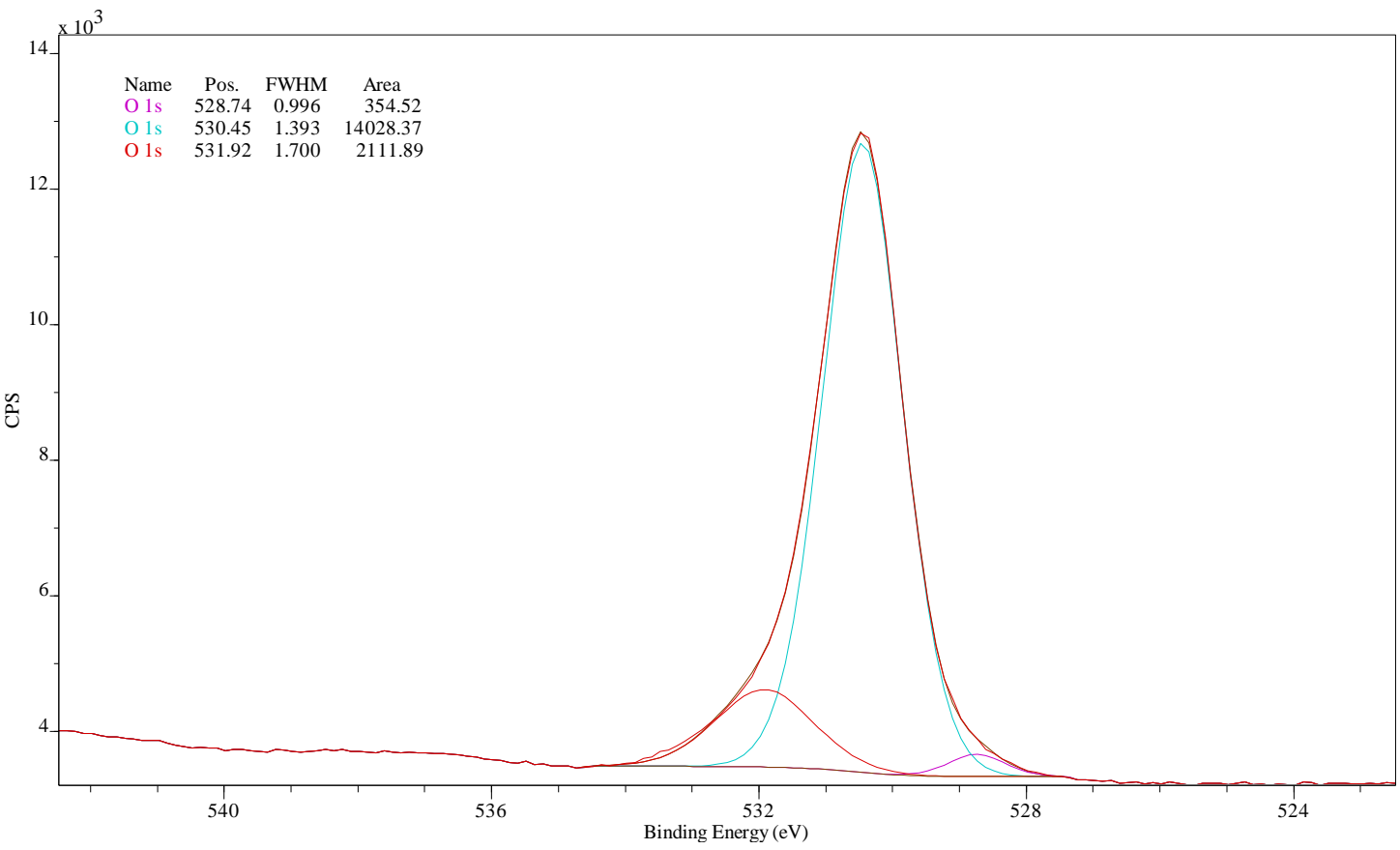

Figure S5. XPS data of $\mathrm{Nb}_{2} \mathrm{O}_{5}-1 \mathrm{~h}$ for (a) Niobium and (b) Oxygen. 
(a)

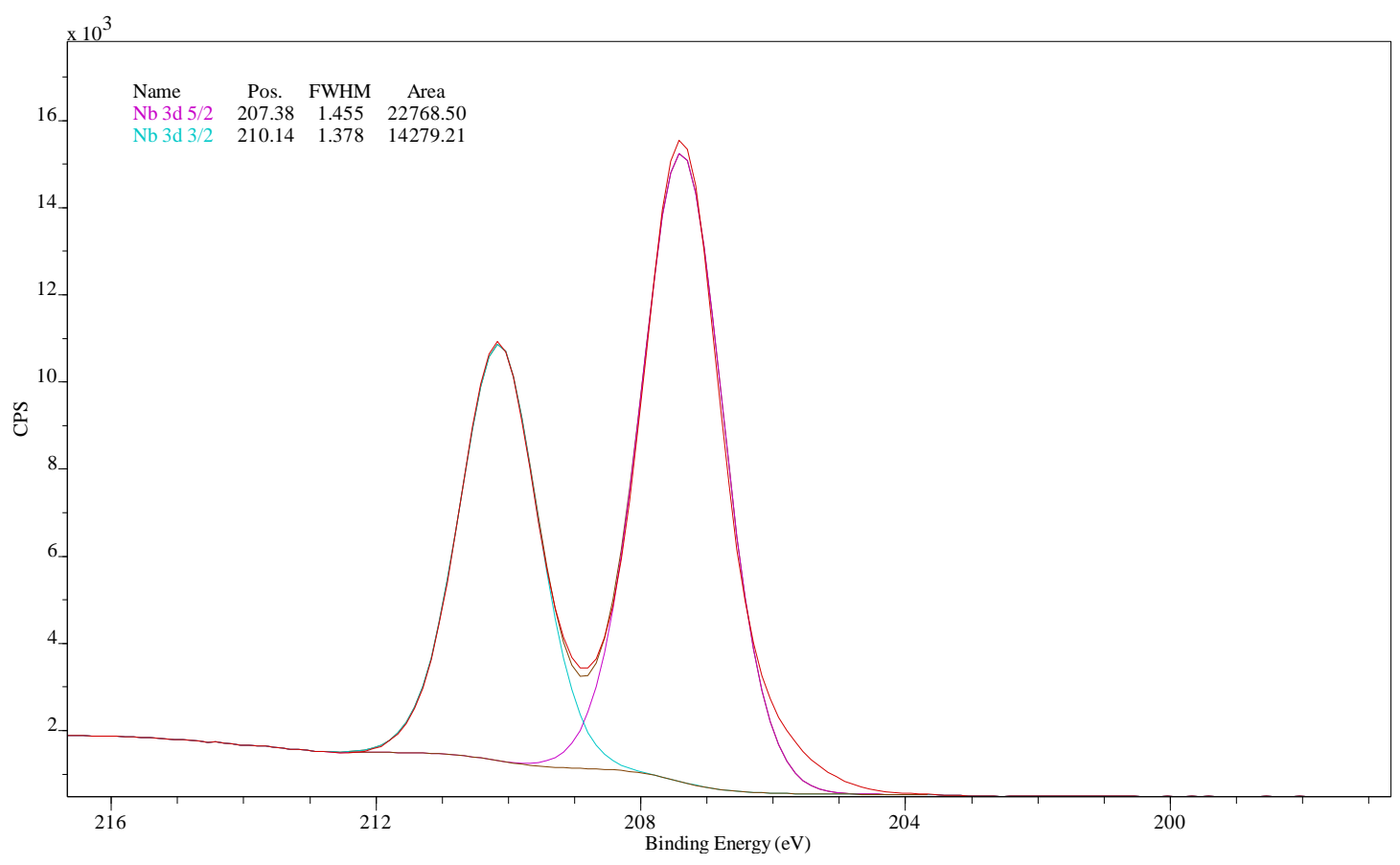

(b)

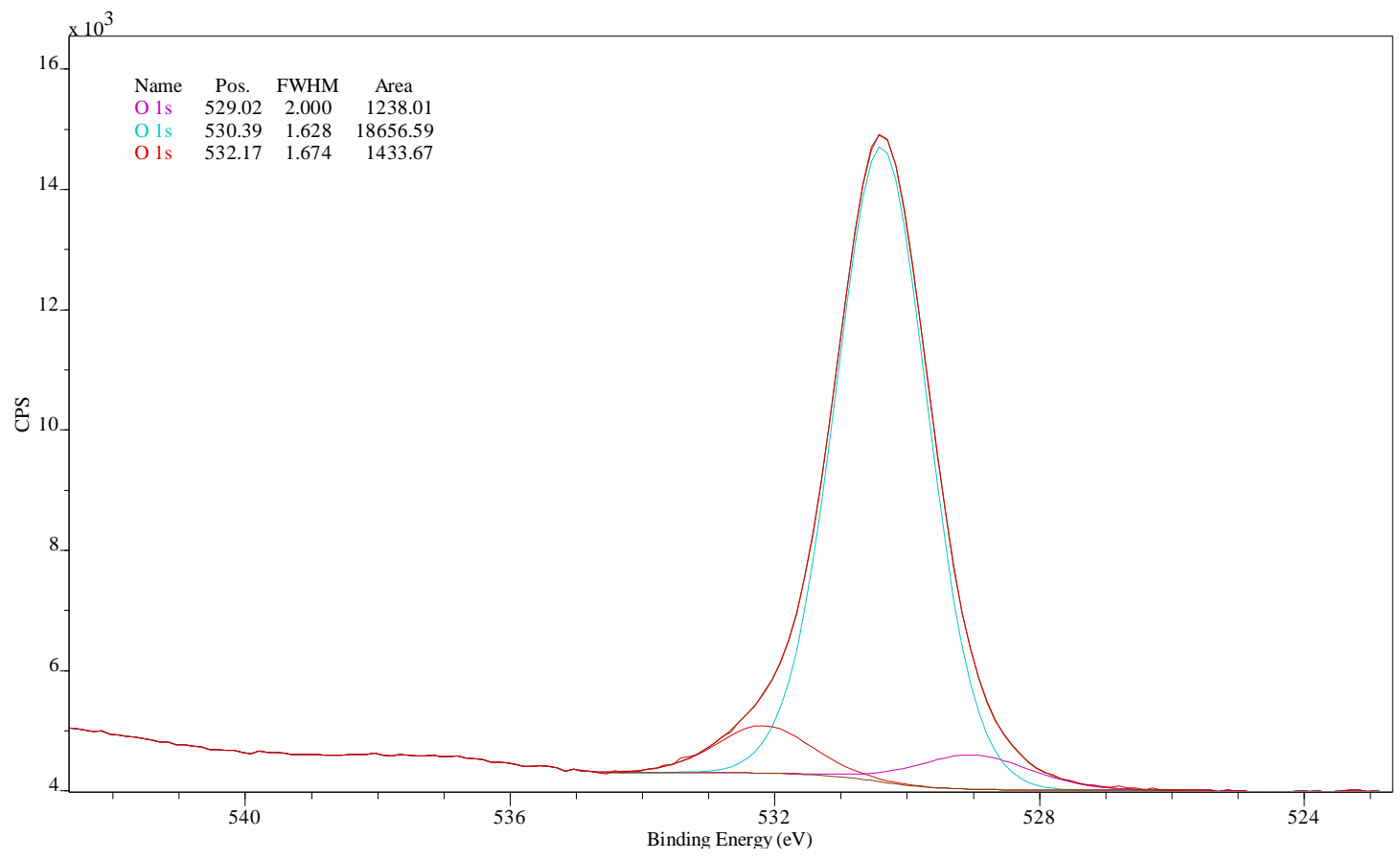

Figure S6. XPS data of of $\mathrm{Nb}_{2} \mathrm{O}_{5}-3 \mathrm{~h}$ for (a) Niobium (b) Oxygen. 


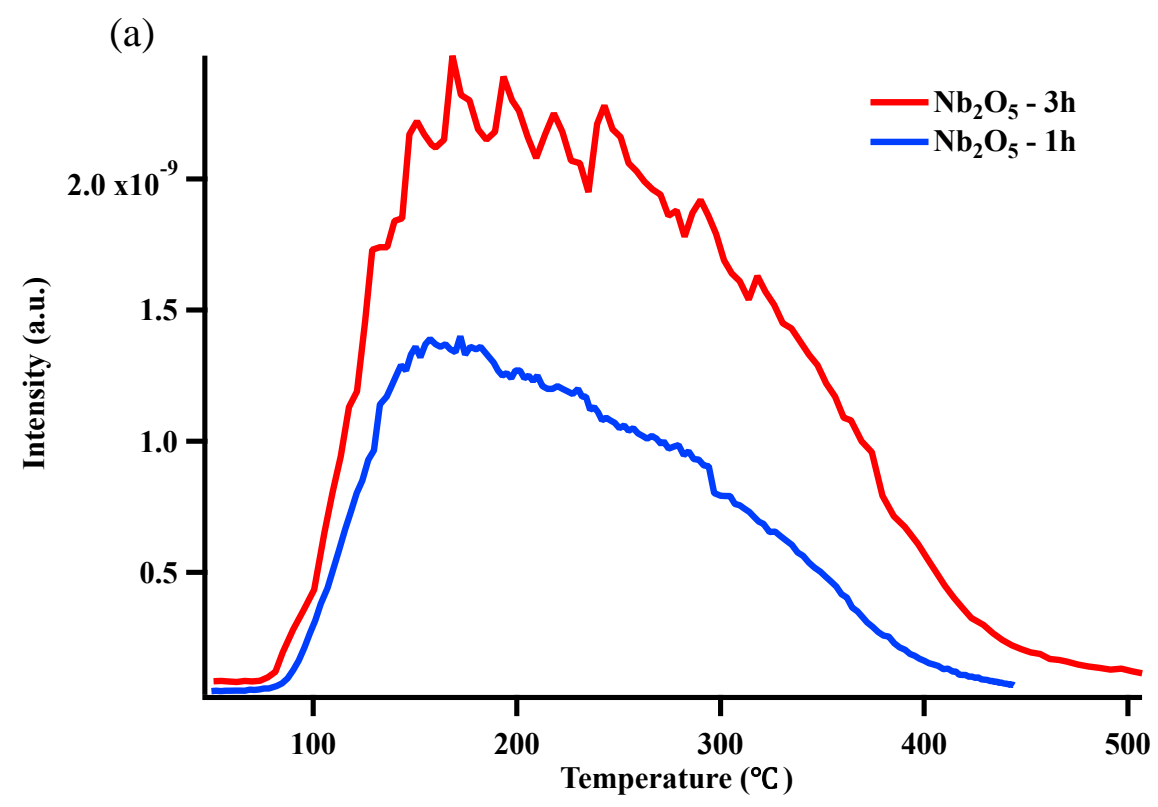

(b)

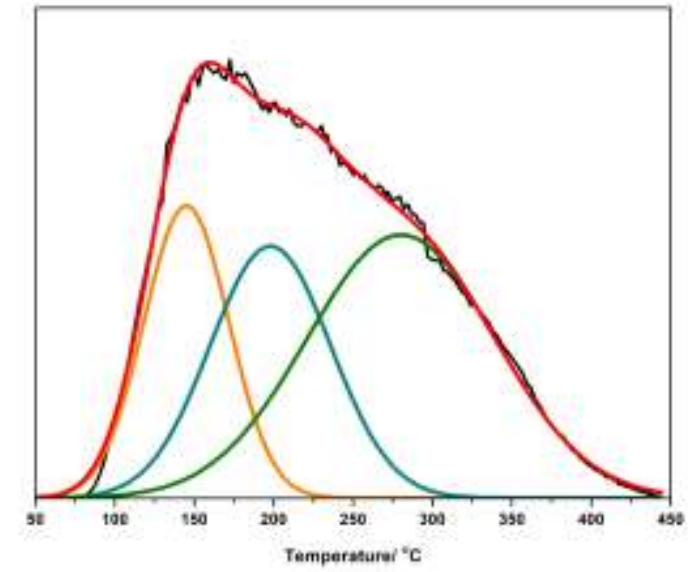

(c)

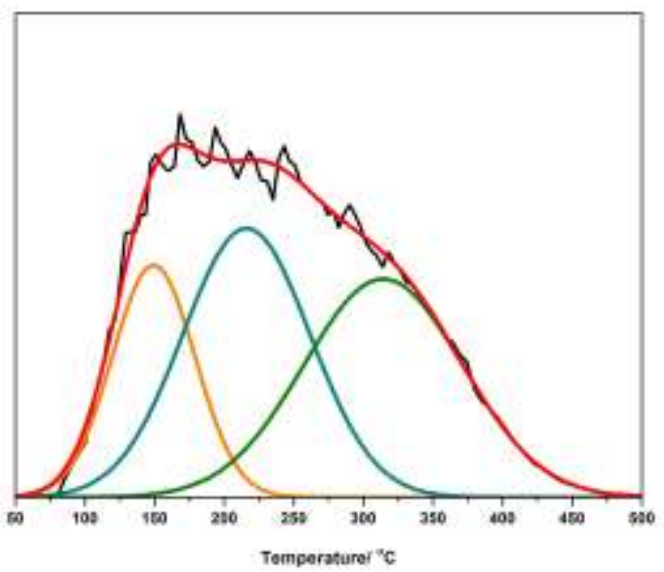

Figure S7. (a) $\mathrm{NH}_{3}-\mathrm{TPD}$ profiles of $\mathrm{Nb}_{2} \mathrm{O}_{5}-1 \mathrm{~h}$, and $\mathrm{Nb}_{2} \mathrm{O}_{5}-3 \mathrm{~h}$, (b) deconvoluted peaks of $\mathrm{Nb}_{2} \mathrm{O}_{5}-$ $1 \mathrm{~h}$, (c) deconvoluted peaks of $\mathrm{Nb}_{2} \mathrm{O}_{5}-3 \mathrm{~h}$.

Table S3. Acid site densities calculated by neutralization ion-exchange method.

\begin{tabular}{|c|c|c|c|}
\hline Material & $\begin{array}{c}\text { Strong acid site } \\
\text { density }(\mathrm{mmol} / \mathrm{g})\end{array}$ & $\begin{array}{c}\text { Weak/moderate acid } \\
\text { site density }(\mathrm{mmol} / \mathrm{g})\end{array}$ & $\begin{array}{c}\text { Total acid site density } \\
(\mathrm{mmol} / \mathrm{g})\end{array}$ \\
\hline $\mathrm{Nb}_{2} \mathrm{O}_{5}-1 \mathrm{~h}$ & 1.4 & 5.2 & 6.6 \\
$\mathrm{Nb}_{2} \mathrm{O}_{5}-3 \mathrm{~h}$ & 1.9 & 8.0 & 9.9 \\
\hline
\end{tabular}


Table S4. Effect of the type of catalyst on alkyne hydration.

\begin{tabular}{|c|c|c|}
\hline Catalyst & Conversion (\%) & Selectivity (\%) \\
\hline $\mathrm{Nb}_{2} \mathrm{O}_{5}-1 \mathrm{~h}$ & 64 & 98 \\
$\mathrm{Nb}_{2} \mathrm{O}_{5}-3 \mathrm{~h}$ & 78 & 97 \\
\hline
\end{tabular}

Reaction conditions: Phenylacetylene $(0.5 \mathrm{mmol})$, catalyst $(20 \mathrm{mg})$, water $(10 \mathrm{mmol})$, toluene (2 $\mathrm{mL}), 100^{\circ} \mathrm{C}, 2 \mathrm{~h}$.

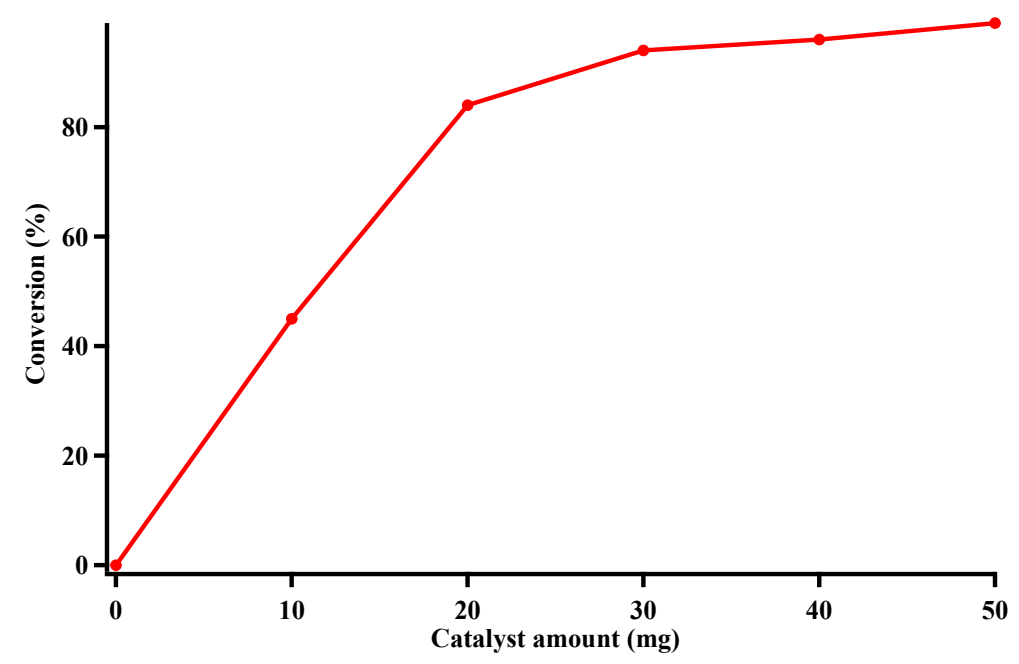

Figure S8. Optimization of catalyst loading.

Reaction conditions: Phenylacetylene $(0.5 \mathrm{mmol})$, water $(10 \mathrm{mmol})$, toluene $(2 \mathrm{~mL}), 100^{\circ} \mathrm{C}, 2 \mathrm{~h}$.

Table S5. Turn over frequencies (TOF) at each catalyst loading.

\begin{tabular}{|c|c|}
\hline Catalyst loading $(\mathrm{mg})$ & TOF $\left(\mathrm{h}^{-1}\right)$ \\
\hline 0 & 0 \\
10 & 1.14 \\
20 & 1.06 \\
30 & 0.79 \\
40 & 0.61 \\
50 & 0.5 \\
\hline
\end{tabular}




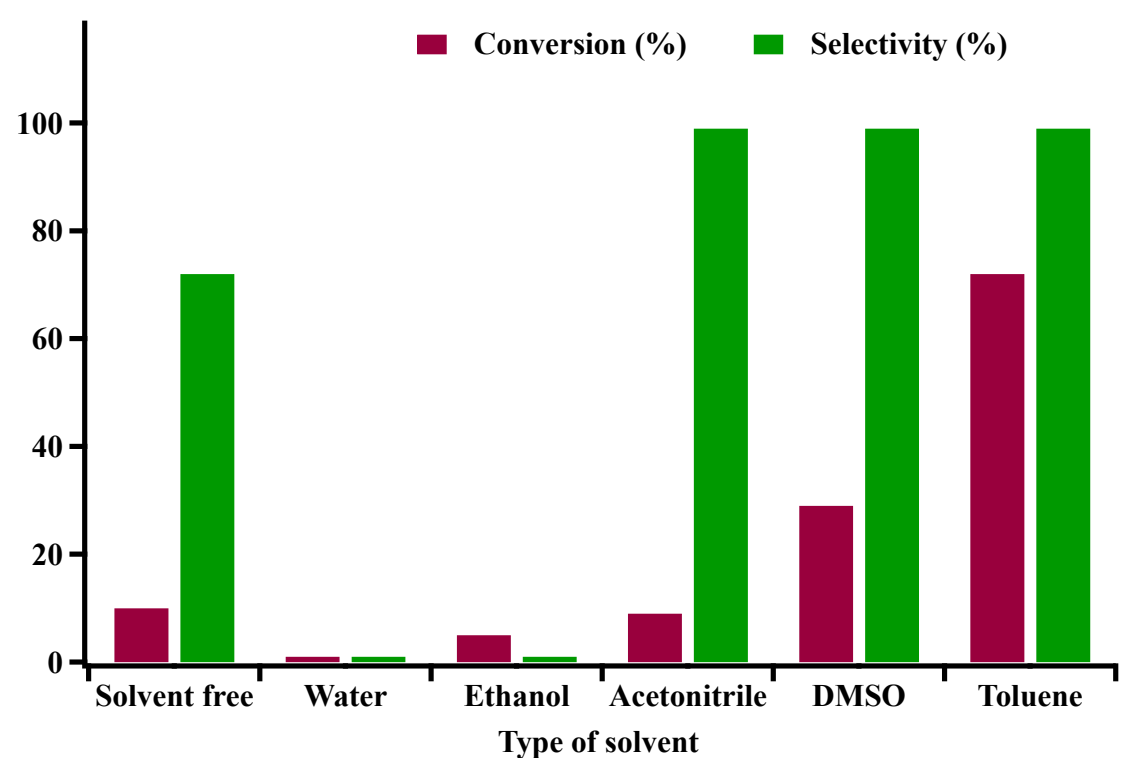

Figure S9. Optimization of the type of solvent.

Reaction conditions: Phenylacetylene $(0.5 \mathrm{mmol})$, water $(10 \mathrm{mmol})$, catalyst $(10 \mathrm{mg})$, solvent (3 $\mathrm{mL}), 100^{\circ} \mathrm{C}, 2.5 \mathrm{~h}$.

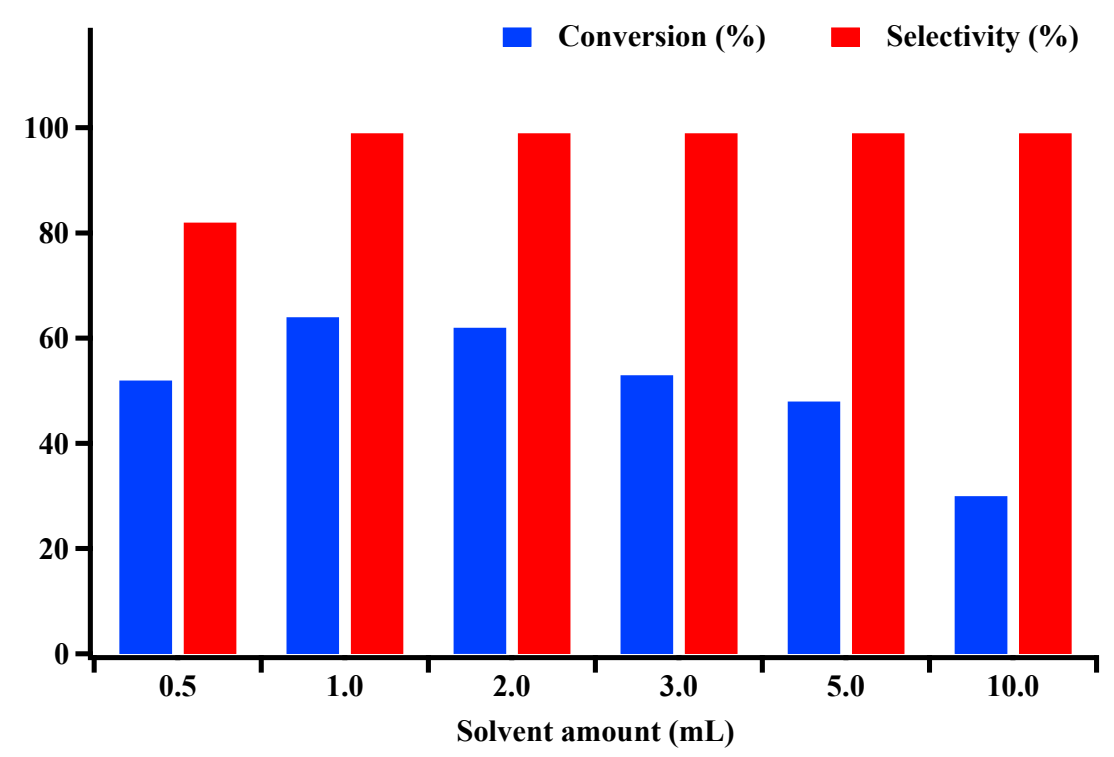

Figure S10. Optimization of the solvent amount.

Reaction conditions: Phenylacetylene $(0.5 \mathrm{mmol})$, water $(10 \mathrm{mmol})$, catalyst $(10 \mathrm{mg}), 100^{\circ} \mathrm{C}, 2$ h. 


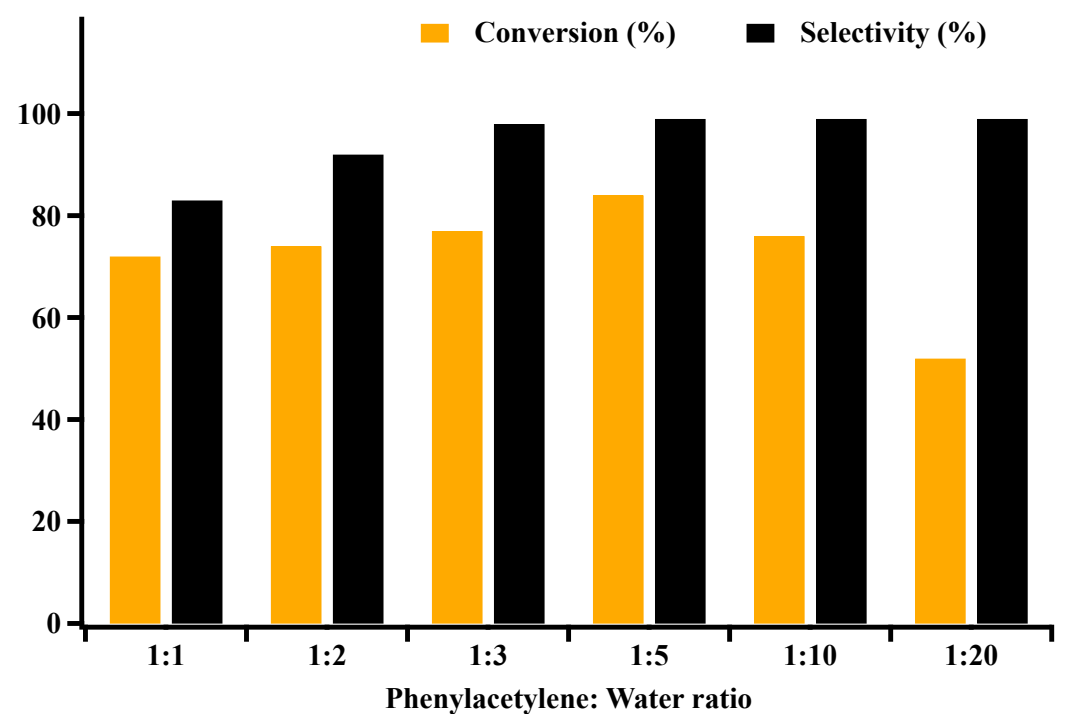

Figure S11. Optimization of the molar ration between starting materials.

Reaction conditions: Phenylacetylene $(0.5 \mathrm{mmol})$, catalyst $(10 \mathrm{mg})$, toluene $(1 \mathrm{~mL}), 100^{\circ} \mathrm{C}, 2 \mathrm{~h}$.

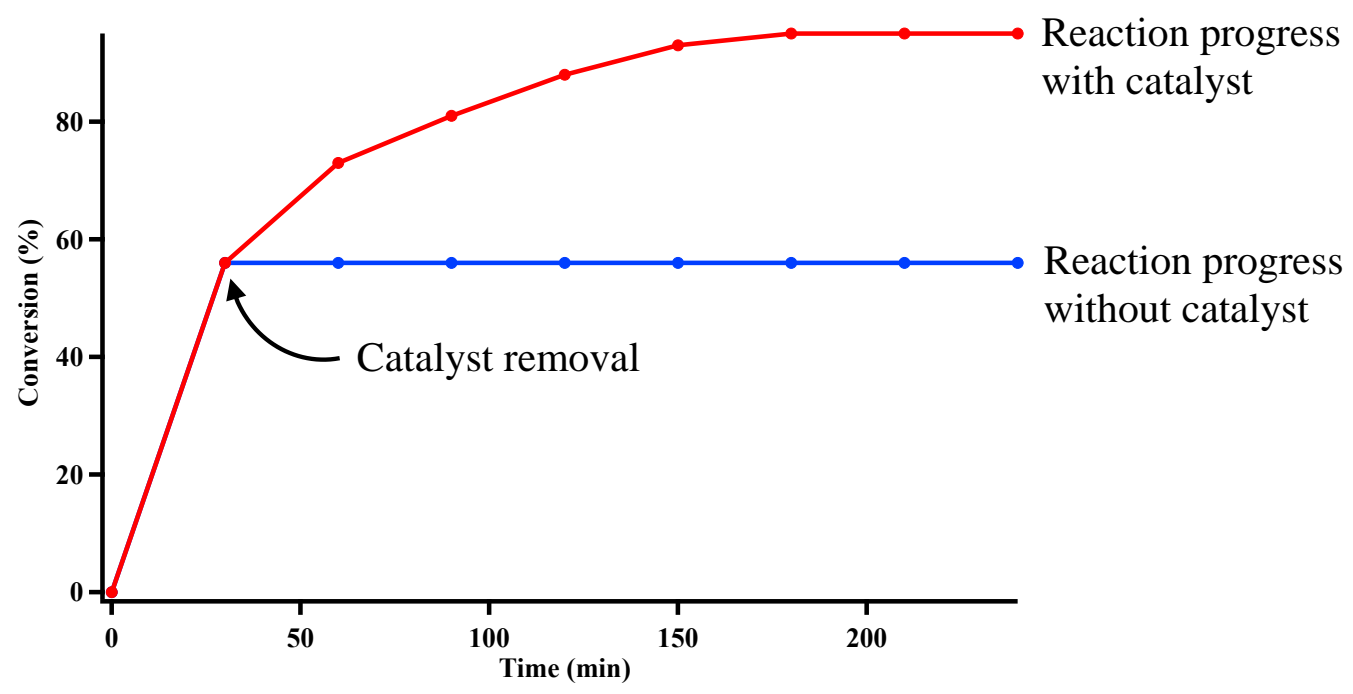

Figure S12. Catalyst leach test for alkyne hydration reaction.

Reaction conditions: Phenylacetylene $(0.5 \mathrm{mmol})$, catalyst $(10 \mathrm{mg})$, toluene $(1 \mathrm{~mL}), 100^{\circ} \mathrm{C}$. 


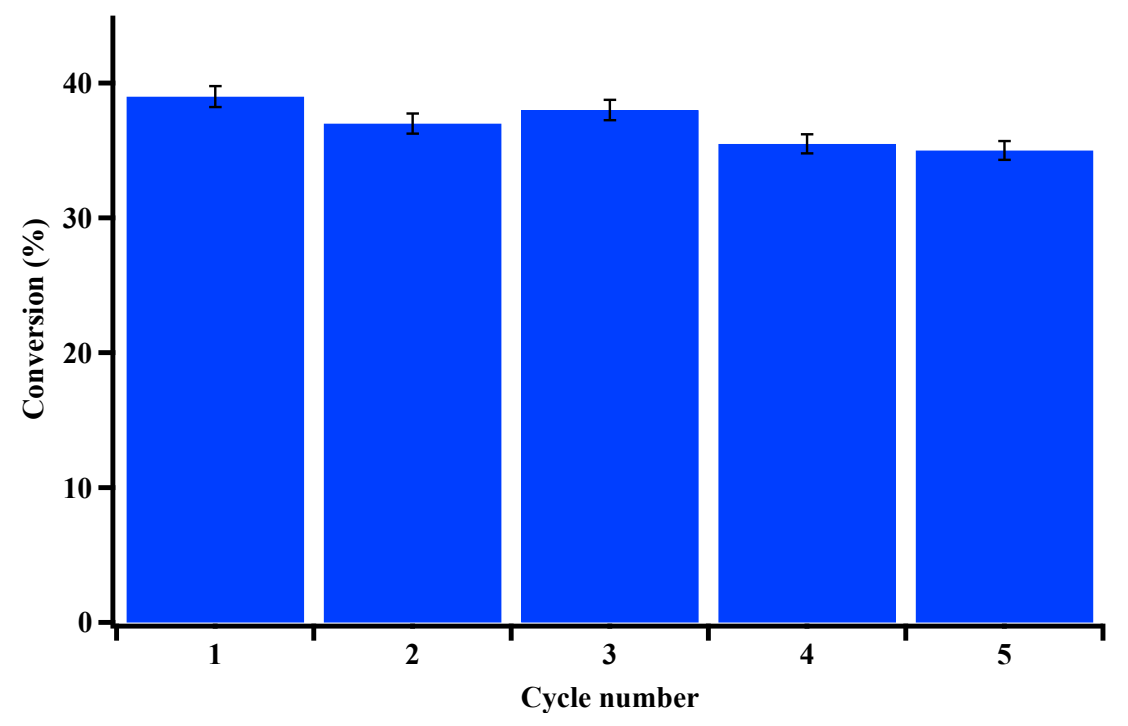

Figure S13. Catalyst reusability test for alkyne hydration reaction.

Reaction conditions: Phenylacetylene $(0.5 \mathrm{mmol})$, catalyst $(10 \mathrm{mg})$, toluene $(1 \mathrm{~mL}), 100^{\circ} \mathrm{C}$.

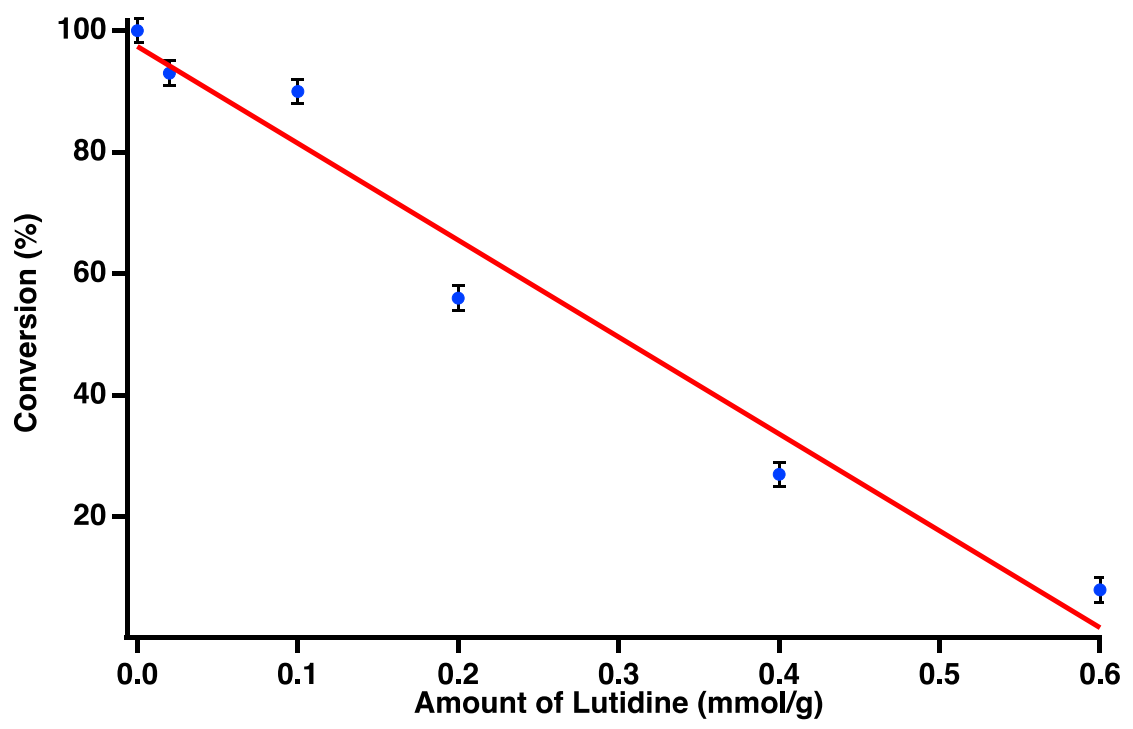

Figure S14. Effect of 2,6-lutidine on the catalyst acid sites. 


\section{1-Phenylethanone}

${ }^{1} \mathrm{H}-\mathrm{NMR}\left(400 \mathrm{MHz} ; \mathrm{CDCl}_{3}\right): \delta 2.61\left(\mathrm{~s}, 3 \mathrm{H}, \mathrm{CH}_{3}\right), 7.46(\mathrm{~m}, J=7.5 \mathrm{~Hz}, 2 \mathrm{H}$, aromatic), $7.56(\mathrm{~m}, J$ $=2.2 \mathrm{~Hz}, 1 \mathrm{H}$, aromatic $), 7.95(\mathrm{~m}, J=4.2 \mathrm{~Hz}, 2 \mathrm{H}) \mathrm{ppm}$<smiles>CC(=O)c1ccccc1</smiles>

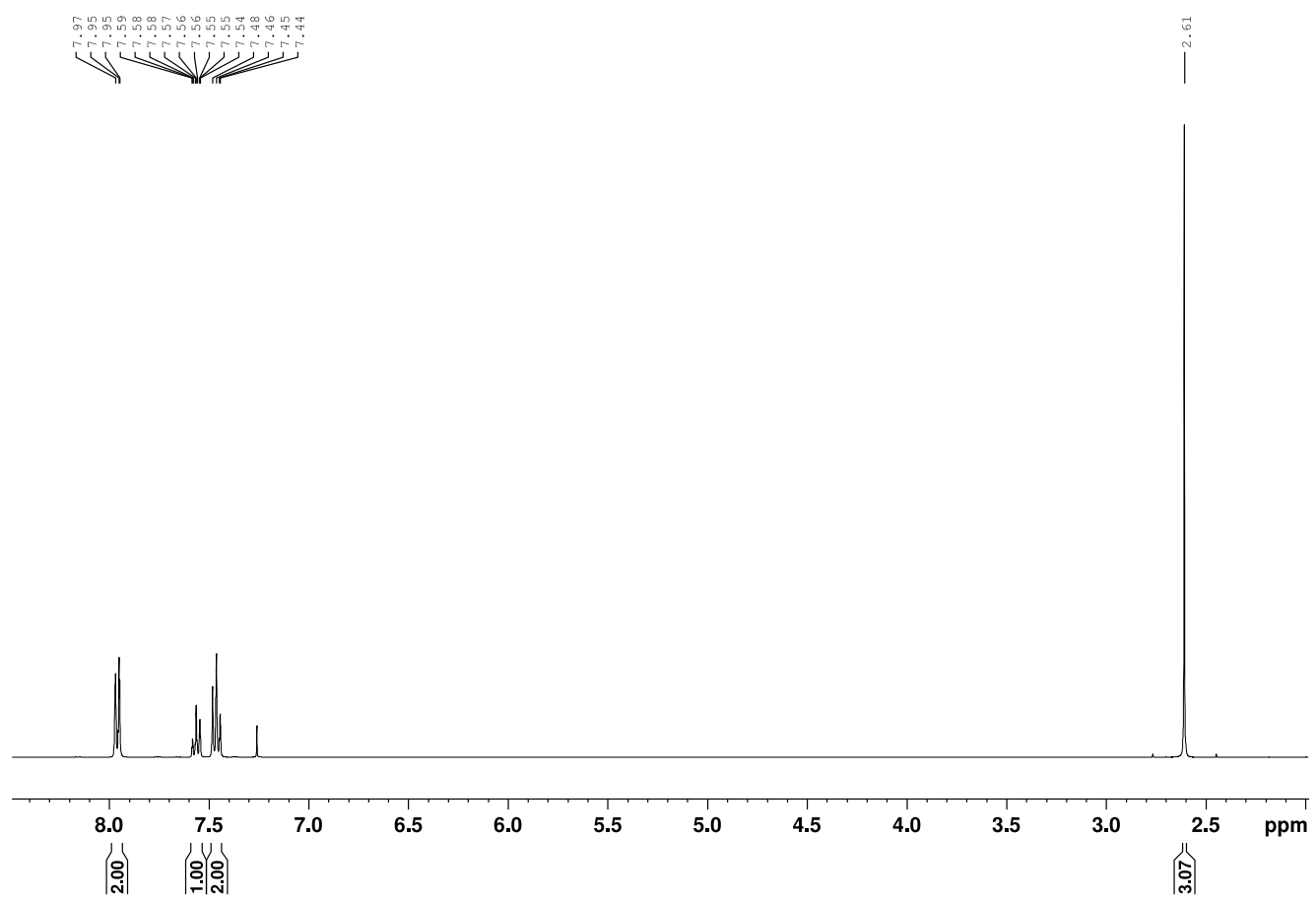

Figure S15. ${ }^{1} \mathrm{H}$ NMR of 1-phenylethanone. 


\section{1-(4-methoxyphenyl)ethenone}

${ }^{1} \mathrm{H}-\mathrm{NMR}\left(400 \mathrm{MHz} ; \mathrm{CDCl}_{3}\right): \delta 2.56\left(\mathrm{~s}, 3 \mathrm{H}, \mathrm{COCH}_{3}\right), 3.87\left(\mathrm{~s}, 3 \mathrm{H}, \mathrm{OCH}_{3}\right), 6.93(\mathrm{~m}, J=8.9 \mathrm{~Hz}$, $2 \mathrm{H}$, aromatic), $7.94(\mathrm{~m}, J=8.9 \mathrm{~Hz}, 2 \mathrm{H}$, aromatic) $\mathrm{ppm}$<smiles>COc1ccc(C(C)=O)cc1</smiles>

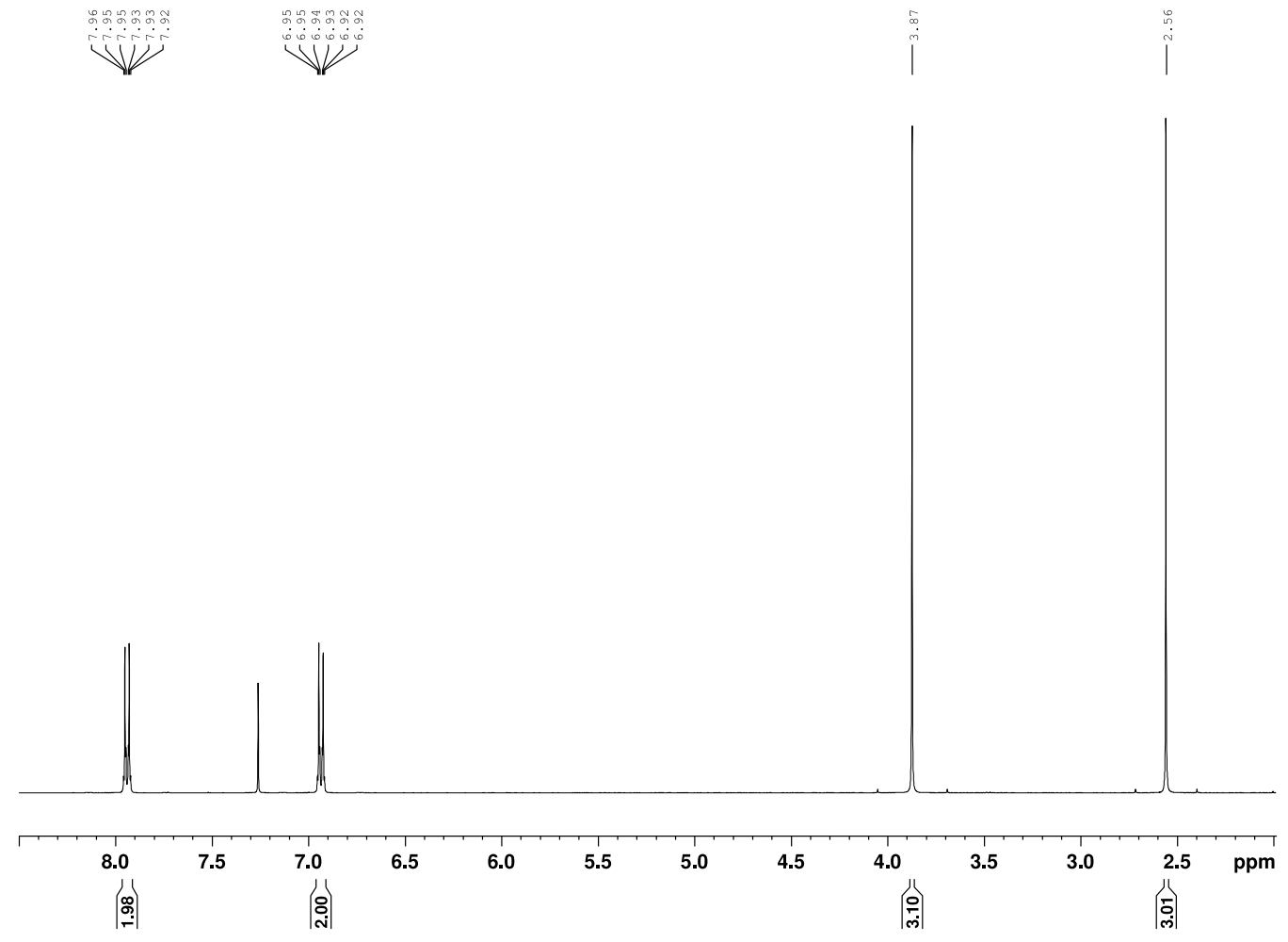

Figure 16. ${ }^{1} \mathrm{H}$ NMR of 1-(4-methoxyphenyl)ethenone. 